\title{
The Effects of 12-Week Physical Exercise Tapping High-level Cog- nitive Functions
}

\author{
Zhiguang $\mathrm{Ji}^{1}$, Tian Feng ${ }^{2}$, and Hongbiao Wang' \\ 'Shanghai University of Medicine and Health Sciences, Shanghai, China \\ 2 Physical Education College of Zhengzhou University, Zhengzhou, China
}

ABSTRACT

The purpose of this study was to investigate the effects of physical exercise tapping high-level cognitive functions on both cognitive function and fitness in older adults. In total, 96 healthy older adults took part in the study. Participants were randomly assigned to four groups: a cognitive training group (CG), physical exercise group (PG), simultaneous cognitive training and physical exercise group $(C+P G)$, and the healthy control group (HG; received physical exercise materials but did not participate in a cognitive or exercise programme). The outcomes were the changes in the 6-minute walk test (6MWT), timed up-and-go (TUG) test, and the computerized modified Stroop task, which included two conditions, a naming condition (nonexecutive) and an executive condition. The results showed that the dynamic balance, cardiorespiratory endurance, and physical activity levels of the PG and C+PG were significantly better than those of the HG after the 12-week intervention. The times of the TUG test in these two groups were also significantly faster than those in the HG. Additionally, the C+PG exhibited better dual-task function than the PG. Over 12 weeks, the CG, PG, and $C+P G$ demonstrated improved performance of executive function, but only the $C+P G$ showed a general facilitative effect on nonexecutive control. Physical exercise tapping high-level cognitive functions and cognitive training can thus improve executive function more quickly than physical exercise alone. Moreover, physical exercise tapping high-level cognitive functions showed better fitness improvement than cognitive training alone, especially in dynamic balance.

older adults

cognitive function

fitness

physical exercise tapping

high-level cognitive func-

tions

\section{INTRODUCTION}

With the ageing of the body, the organs and physiology of elderly individuals decline, age-related degeneration occurs, and cognitive function, an important indicator of brain function, is also impaired. Cognitive function refers to the ability of the human brain to process, store, and extract information. Cognitive ageing refers to the decline in cognitive function that occurs during ageing, mainly in memory. While multiple aspects of cognitive function have been linked to ageing, the cognitive domain of executive function is particularly relevant. Executive function describes the complex and higher-order cognitive processes used to monitor, manage, and regulate a set of self-directed, purpose-oriented actions to reach a desired goal.

Although it has been confirmed in many studies that exercise can delay age-related cognitive decline (Albinet et al., 2016), the type of exercise leading to the greatest degree of these positive effects remains unclear. While most previous studies used physical exercise, some researchers believe that low cognitive requirements and highly automatic physical exercise have less of an impact on the inhibitory function of elderly people (Huang et al., 2014). Therefore, researchers have begun to pay attention to exercises with high cognitive requirements. León et al. (2015) found that exercises with high cognitive requirements (such as Tai Chi) had a larger impact on cognitive function than exercises with low cognitive requirements (such as walking or cycling). Similarly, Fabre et al. (2002) found that a combination of physical and

Corresponding author: Hongbiao Wang, 279 Zhouzhu Highway, Pudong New Area, Shanghai, 201318, P. R. China. E-mail:wanghb@sumhs.edu.cn 
cognitive exercises led to greater benefits than performing one of the activities alone (Fabre et al., 2002).

Exercise with high cognitive requirements improved not only cognitive, but also executive functions in elderly people. Some studies have shown that Tai Chi can simultaneously improve the performance of the Trail Making Test A and B (TMT-A and TMT-B; (Hung \& Andreas, 2012; Reid-Arndt et al., 2012). In contrast, Matthews and Williams (2008) found that the Tai Chi group improved in TMT-B, but not in TMT-A. The TMT-A and TMT-B tests can be considered to involve different cognitive processing levels, attention and executive functions (Smith et al., 2010). Studies have also shown that Tai Chi improves mental attention and memory, not just executive control (Kim et al., 2016; Man et al., 2010). In a study on physical exercise, the benefits of exercise for cognitive decline were found to be selective rather than universal (Boucard et al., 2012). Specifically, cognitive functions, which are highly dependent on the execution control process of the frontal lobe, are more prone to age-related decline and are also more sensitive to exercise, including inhibition. Thus, many of these features are suitable for physical exercise tapping high-level cognitive functions. Therefore, its specificity of influence has yet to be investigated. A recent review reinforced this idea by using training programmes combined with physical and cognitive activities, as this combination is more beneficial for neuronal recruitment and cognitive function (Curlik 2nd \& Shors, 2013). Zhu et al. (2016) analysed 20 interventional studies including 2667 participants. The results showed that there was no significant difference between the cognitive interventions, but the effects of cognitive function were greater than those of physical exercise. However, current research lacks separate consideration of motor and cognitive factors, making it difficult to determine whether simultaneous exercise and cognitive activity can provide additional cognitive benefits compared with individual exercise or cognitive training.

Inhibition refers to the ability of the brain to suppress an independent stimulus or advantageous response that may interfere with the current task to complete the current task at the time of information processing. The inhibitory function plays an important role in cognitive decline. Hartman and Hasher (1991) put forward the theory of inhibition of cognitive function ageing, according to which the individual can attend to irrelevant information with increasing age, and it is difficult to focus attention on the target information. In a large number of studies, the decline in cognitive function in elderly people is shown to be largely due to the inability to effectively suppress irrelevant information, which is regarded as one of the most important components of execution function. Therefore, related research on the decline in inhibitory function plays a very important role in the study of the deterioration of cognitive function.

On the other hand, although studies have examined sports for physical fitness, there are few studies on physical fitness for different cognitive requirements, especially for those in close association with daily life. For example, dual-task processing capability is clinically important because it is related to reaction time (RT), walking speed, the likelihood of contact with obstacles while walking, and the risk of falls (Pichierri et al., 2011). Age-related declines in executive function may cause distraction and are an important risk factor for falls in older adults (Muir et al., 2012). The current study focused on the improvement of cognitive function in physical exercise by tapping high-level cognitive functions and the assessment of fitness-related functional levels.

This experiment explored the effects of a 12-week programme of physical exercise tapping high-level cognitive functions, physical exercise alone, or cognitive training alone on the cognitive function and fitness of elderly people. In this study, we used a dual-task mode to represent the requirements of physical exercise tapping high-level cognitive functions. We hypothesized that participation in a 12 -week programme of physical exercise tapping high-level cognitive functions would have a significantly higher inhibitory effect on the elderly than on other groups, and that the effect of physical exercise tapping high-level cognitive functions on physical fitness would be similar to that of physical exercise, although it appears earlier.

\section{MATERIALS AND METHODS}

\section{Participants}

This experiment recruited 162 healthy elderly individuals aged 65 to 70 years from a community in Shanghai. Participants were requested to have no regular exercise habits in the past year (the frequency of exercise was less than twice a week) and to not regularly participate in any high-level cognitive activities, such as mah-jong, chess, or card activities. Additional inclusion criteria included age between 60 and 70 years, ability to walk for at least 5 minutes unassisted, and a Mini-Mental State Examination (MMSE) score $>26$. Through a preliminary questionnaire survey based on the above criteria, 100 elderly people were ultimately included in the experiment. Participants were randomly assigned to four groups: a healthy control group (HG), cognitive training group (CG), physical exercise group (PG), and simultaneous cognitive and physical exercise group (C+PG). Among the subjects, four withdrew from the experiment for personal reasons. Therefore, 96 subjects completed the intervention experiment. The basic information of the participants is shown in Table 1.

\section{Experimental Procedure}

Each participant was asked to participate in a questionnaire before the formal experiment. The participants signed the consent form and completed the following questionnaires: health history and demographic questionnaires, physical activity questionnaire (International Physical Activity Questionnaire, IPAQ, Hallal \& Victora, 2004), exercise habit questionnaire, and the Mini Mental State Examination (MMSE, Cockrell \& Folstein, 1988). The total of 100 elderly subjects who met the experimental requirements were randomly divided into the four groups and subjected to cognitive tests and fitness assessments before, during, and after the intervention. The experimental flow chart is shown below in Figure 1.

\section{Intervention}

The intervention lasted for 12 weeks. According to the recommendations of the American College of Sports Medicine (Brent, 2009), the 


\begin{tabular}{lcccccc}
\cline { 2 - 6 } & $\begin{array}{l}\text { TABLE 1. } \\
\text { Participant Characteristics }\end{array}$ & & & \\
\hline & $\begin{array}{c}\text { C+PG }(\boldsymbol{n}= \\
\mathbf{2 4})\end{array}$ & $\mathbf{P G}(\boldsymbol{n}=\mathbf{2 5})$ & $\mathbf{C G}(\boldsymbol{n}=\mathbf{2 2})$ & HG $(\boldsymbol{n}=\mathbf{2 5})$ & $\boldsymbol{F ( 3 , 9 2 )}$ & $\boldsymbol{p}$ \\
\hline Age, years & $65.45 \pm 4.40$ & $66.52 \pm 5.25$ & $65.53 \pm 4.32$ & $66.34 \pm 3.33$ & 0.81 & .50 \\
Gender (women, $n)$ & 14 & 13 & 12 & 14 & 0.41 & .74 \\
Weight, kg & $59.52 \pm 5.32$ & $57.68 \pm 5.12$ & $58.71 \pm 6.89$ & $57.85 \pm 5.12$ & 0.41 & .75 \\
Height, cm & $163.79 \pm 6.42$ & $162.04 \pm 5.97$ & $162.21 \pm 7.89$ & $161.43 \pm 7.46$ & 1.07 & .37 \\
BMI & $22.31 \pm 2.45$ & $22.75 \pm 1.43$ & $22.21 \pm 1.54$ & $22.11 \pm 1.53$ & 0.42 & .73 \\
MMSE & $27.54 \pm 0.53$ & $27.21 \pm 0.65$ & $27.23 \pm 0.52$ & $27.43 \pm 0.42$ & 1.20 & .32 \\
Education, years & $10.21 \pm 2.43$ & $11.53 \pm 2.47$ & $10.21 \pm 2.35$ & $10.12 \pm 2.43$ & 0.62 & .60 \\
\hline
\end{tabular}

Note. $\mathrm{C}+\mathrm{PG}=$ Simultaneous cognitive and physical exercise group; $\mathrm{PG}=$ Physical exercise group; $\mathrm{CG}=$ Cognitive training group; $\mathrm{HG}=$ Healthy control group.

intervention group had three classes per week, lasting approximately 60 minutes each class. Each group had two experimenters to guide and supervise the activities.

The HG did not participate in any intervention activities and received weekly physical exercise materials and a health exercise lecture every four weeks.

The curriculum of the CG was designed to improve executive function and memory. Specific courses included verbal fluency tests, mental arithmetic tasks, visual search tasks, and reverse passwords. Participants completed all cognitive interventions at the indoor activity centre. The verbal fluency test training allowed the participants to recall as many words as possible, including vegetables, fruits, countries, sports, occupations, and so forth (Hartman \& Hasher, 1991). The mental arithmetic tasks required the subject to perform continuous addition or subtraction as required (Shinya \& Ryuta, 2008). Visual search tasks required the current scene features of the subject to be described as required (Gates et al., 2011). Reverse password practice allowed the subject to respond to the opposite of the password, including raising the right arm - left arm, raising the right leg - left leg, closing the right eye - left eye, sitting - standing, turning right - turning left, looking forward - looking backwards, looking up - bowing down, and so forth.

The PG was required to complete the exercise (treadmill walking) at the activity centre. Each exercise included a 10-minute warm-up and a 10-minute cool-down. The intensity of the exercise was maintained at $55 \%-60 \%$ of the heart rate reserve. The monitoring method used a polar heart rate monitoring system.

The C+PG simultaneously performed cognitive training and physical exercise. For example, participants performed a verbal working memory task while simultaneously walking on the treadmill.

\section{Anthropometrics and Fitness}

Height (in meters) and body weight (in kilograms) were used to calculate the body mass index $\left(\mathrm{BMI}, \mathrm{kg} / \mathrm{m}^{2}\right)$. Hand-grip strength was measured with a hand-held dynamometer (Jamar; Sammons Preston

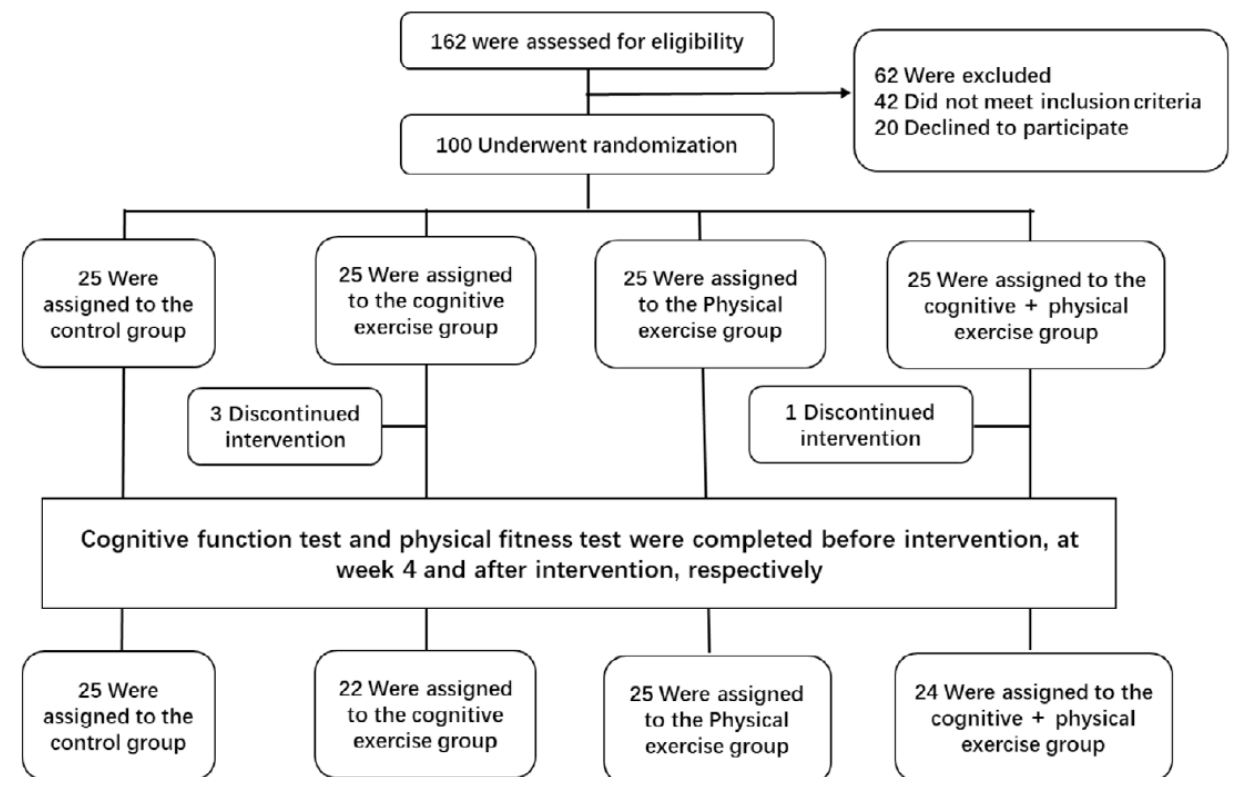

FIGURE 1.

The experimental flowcharts. 
Naming condition

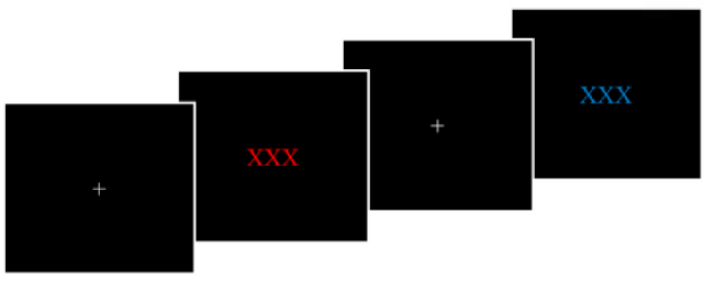

Executive condition

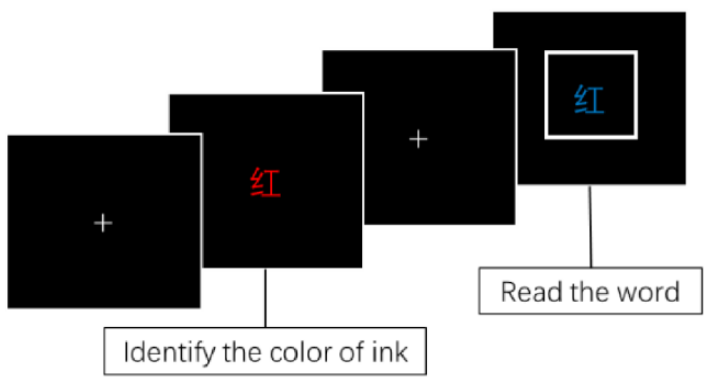

FIGURE 2.

Illustration of the modified Stroop task.

Rolyan; Nottinghamshire, UK). Participants performed grip tests twice on each hand and analysed them with the best results. The level of physical activity was assessed using the Taiwanese version of the IPAQ (Liou et al., 2008). The short version of the IPAQ is an eight-item scale that estimates the number of minutes of high-intensity and moderateintensity activities, walking, and sedentary behaviour over the past seven days.

The 6-minute walk test (6MWT) is widely used to assess cardiorespiratory fitness (Burr et al., 2011; Camarri et al., 2006). The participants are instructed to "cover as much distance as possible" during the test. The number of metres walked is recorded.

The timed up-and-go (TUG) test was used to evaluate dynamic balance (Clark et al., 2010; Porciuncula et al., 2016). The participants are instructed to stand up from a seated position and walk to a marked post $3 \mathrm{~m}$ away as quickly and safely as possible, and then turn around, walk back to the chair and sit. The study selected the following two conditions for the dual-task TUG test: (a) in the mTUG task, remember six random numbers while walking and recall the correct numbers after sitting down, and (b) in the cTUG task, go across a $17 \mathrm{~cm}$ high (kerb height) obstacle while walking. The tests were completed three times, and the shortest duration for each task was recorded (Shumway-Cook et al., 2000).

\section{Modified Stroop Task}

The computerized modified Stroop task included the following two conditions: a naming condition (nonexecutive) and an executive condition (Bohnen et al., 1992; Dupuy et al., 2015). All the experimental trials began with a fixation cross for $1 \mathrm{~s}$, and all visual stimuli appeared at the centre of the computer screen for a duration of $2 \mathrm{~s}$. The participants provided their responses by pressing the "F" button for "blue" and the "J" button for "red". Each condition included two blocks, and each block was composed of 30 trials, resulting in 120 trials for the two experimental conditions. In the naming condition, a visual stimulus (the letters "XXX") coloured red or blue was presented, with 15 trials for each colour. Half of the trials were the same as the original Stroop task, in which the response was the colour of the word (inhibition). In the other trials, the participants were instructed to read the word and ignore the colour of the writing (switch).
The executive condition included inhibition and switch. Thirty trials (20 inhibition and 10 switch) were included in one block of the executive condition (see Figure 2). The experimental blocks were interspersed with a 1 min condition called "rest." The total Stroop task duration was approximately 15 minutes.

\section{Statistical Analyses}

A one-way analysis of variance (ANOVA) was used to analyse age, weight, height, BMI, MMSE score, and education level. A $4 \times 3$ (Group [HG, CG, $\mathrm{PG}$, and $\mathrm{C}+\mathrm{PG}] \times 3$ Time [baseline, midpoint, and post-intervention]) mixed-design ANOVA was used to analyse grip strength, 6MWT, and IPAQ scores among the four groups. A $4 \times 3 \times 3$ (Group [HG, CG, $\mathrm{PG}$, and $\mathrm{C}+\mathrm{PG}] \times$ Time [baseline, midpoint, and post-intervention] $\times$ Conditions [TUG, mTUG, and cTUG tests]) mixed design was used to analyse group, time, and TUG test interactions.

E-Prime (version 1.1) software was used to collect the RT data (i.e., the elapsed time from the presence of the test stimulus to the press of the button) and the correct rate data. All erroneous trials and trials with more than three SDs were considered outliers and removed prior to analysis. A $4 \times 3 \times 2$ (Group [HG, CG, PG, and C+PG] $\times$ Time [baseline, midpoint, and post-intervention] $\times 2$ Conditions [non-executive conditions, and execution conditions]) mixed design was used to analyse the RT and correct rate in the Stroop task and numbers comparing tasks for each group.

Post hoc comparisons were performed using Fisher's least significant difference (LSD) to test differences between groups. The magnitude of the effect is expressed as a deviation of $\eta 2$ to assess the effects when main and interaction effects reach significant levels. The significance threshold was set to $p<0.05$.

\section{RESULTS}

\section{Grip Strength, 6-minute Walk Test, and Physical Activity Level}

No significant main effects of time, $F(2,184)=0.22, p=.81$, or group, $F(3,92)=0.13, p=.95$, were found in grip strength. No interaction effect 

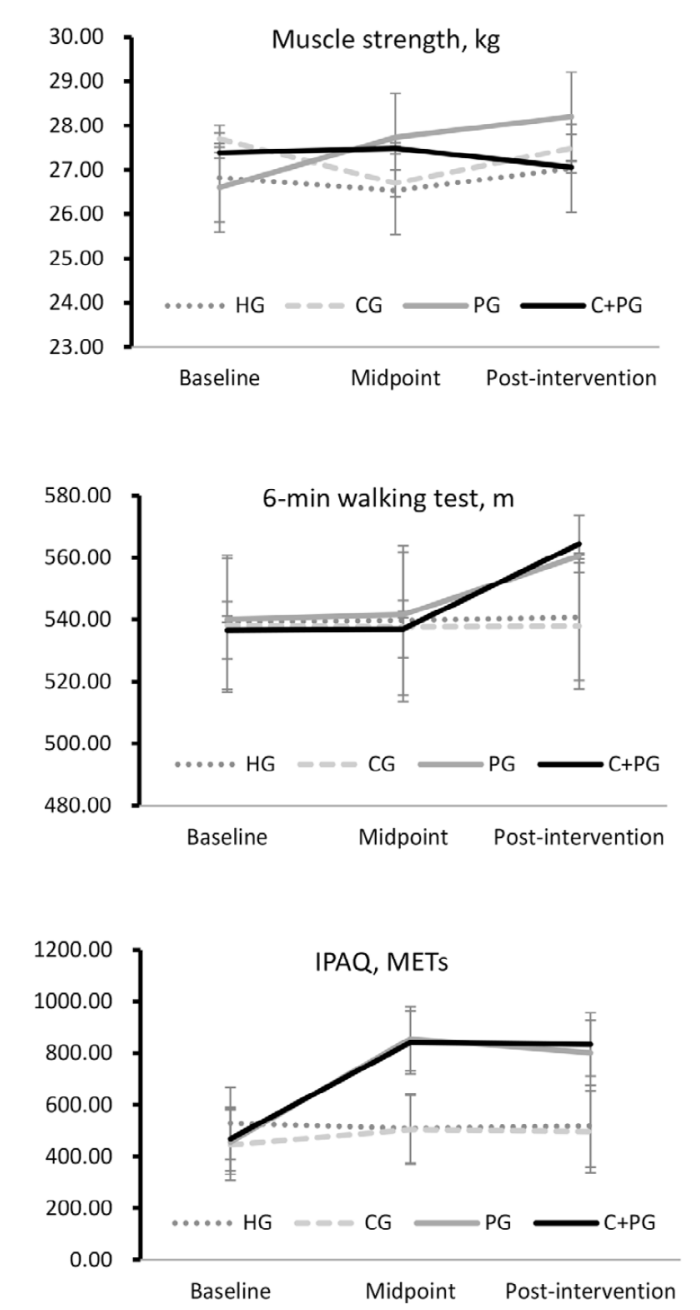

\section{FIGURE 3.}

The results of the grip strength, 6-minute walk test, and physical activity level.

was found between time and group, $F(6,184)=0.46, p=.84$. In the 6MWT, there was a significant main effect of time, $F(2,184)=15.49, p<$ $.01, \eta^{2}=.14$, and interaction between time and group, $F(6,184)=4.89, p$ $<.01, \eta^{2}=.14$, but no main effect of group, $F(3,92)=1.91, p=.13$. The main effects of time, $F(2,184)=20.53, p<0.01, \eta^{2}=.18$, and group, $F(3$, $92)=11.47, p<.01, \eta^{2}=0.27$, and the interaction of time $\times$ group, $F(6$, $184)=5.77, p<0.01, \eta^{2}=.16$, were also found for physical activity. Post hoc analysis showed that at baseline, the HG was greater than the CG $(p<.05)$, the $\mathrm{C}+\mathrm{PG}$ and PG were greater than the HG $(p<.01)$ in the middle test, and the $\mathrm{C}+\mathrm{PG}$ and $\mathrm{PG}$ were greater than the $\mathrm{HG}(p<.01)$ in the post-test. All results are presented in Figure 3.

\section{The Timed Up-and-Go Test}

The TUG test results show (see Figure 4 ) that the main effects of time, $F(2,184)=3.91, p<.05, \eta^{2}=.04$, and conditions, $F(2,184)=69.65, p<$ $.01, \eta^{2}=.43$, were significant, but there was no significant main effect of group, $F(3,92)=1.08, p=0.36$. The interaction of time $\times$ condition $\times$ group, $F(12,372)=2.8, p<.01, \eta^{2}=.08$, was significant. Post hoc analyses indicated that the $\mathrm{C}+\mathrm{PG}$ had faster times than the HG and CG $(p<.05)$, and both the PG and CG had faster times than the HG $(p<.05)$ in the
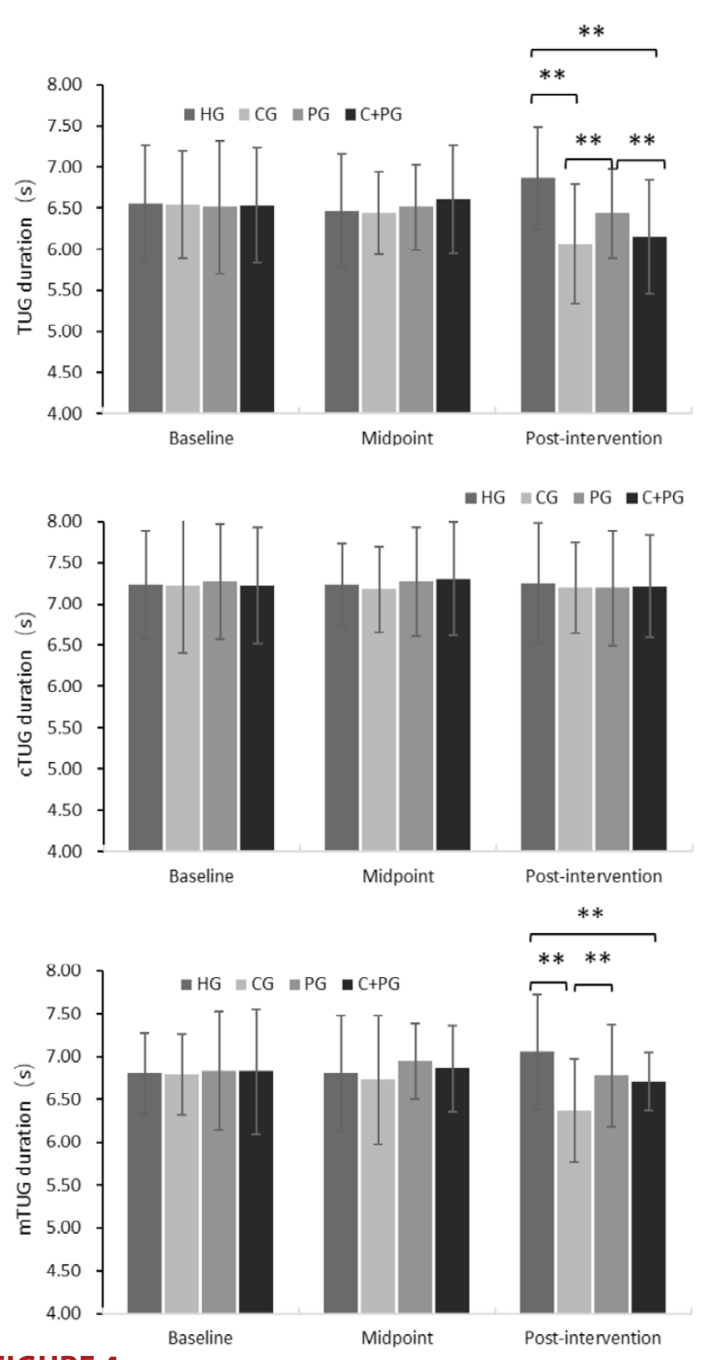

FIGURE 4.

Changes in the timed up-and-go test during the interventions.

TUG test. There was no significant difference between the four groups at the baseline and midpoint, but at the post-intervention, the $\mathrm{C}+\mathrm{PG}$ was found to be significantly faster than all the other groups $(p<.05)$, and the PG was faster than the HG $(p<.05)$ on the mTUG test.

\section{Stroop Task}

When RT was considered, the results show that the main effects of time, $F(2,184)=8.01, p<.01, \eta^{2}=.08$, task, $F(1,92)=1383.07, p<.01, \eta^{2}=.94$, and group, $F(3,92)=6.52, p<.01, \eta^{2}=.13$, were significant and that the interactions of time $\times$ task $\times$ group, $F(6,184)=2.49, p<.05, \eta^{2}=.08$, and time $\times$ group, $F(6,184)=5.89, p<.01, \eta^{2}=.16$, were significant. The interactions of time $\times$ task, $F(2,184)=2.21, p=.11$, and task $\times$ group, $F(3$, $92)=0.28, p=.84$, were nonsignificant. Post hoc analyses indicated that the PG had shorter post-test RT than that at baseline on the executive task $(p=.46)$. The $\mathrm{CG}$ had a shorter post-test $\mathrm{RT}$ than the RT at baseline on the naming task $(p<.05)$. The $\mathrm{C}+\mathrm{PG}$ group had a shorter post-test RT than the RT at baseline on both tasks $(p<.05)$.

There were no main effects of task, $F(1,92)=42.91, p<.01, \eta^{2}=.36$, time, $F(2,184)=0.33, p=.72$, or group, $F(3,92)=0.12, p=.97$, on the correct rate. The interactions of time $\times$ task $\times$ group, $F(6,184)=0.15, p=$ 

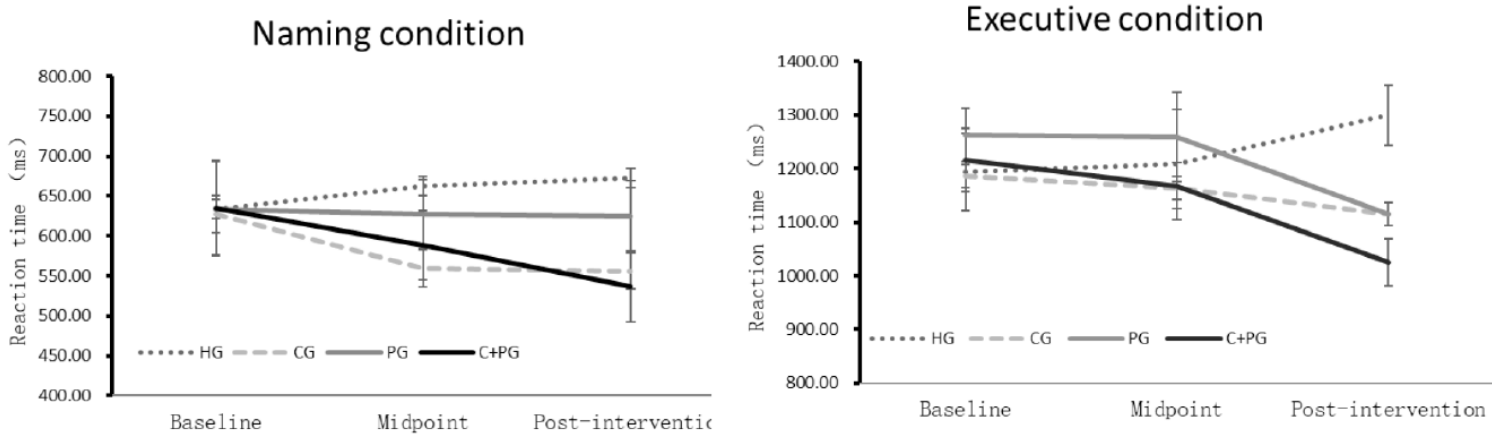

FIGURE 5.

Reaction times in the modified Stroop task.

.99 , time $\times$ group, $F(6,184)=0.19, p=.99$, time $\times$ task, $F(2,184)=0.22$, $p=.81$, and task $\times$ group, $F(3,92)=0.12$, $=.98$ were also nonsignificant.

\section{DISCUSSION}

The purpose of this study was to investigate the effects of physical exercise tapping high-level cognitive functions on cognitive function and fitness in older adults. The results of the study showed that 12 weeks of training in physical exercise alone, high-level cognitive physical exercise, or cognitive training alone can improve executive function, which is consistent with previous studies (Law et al., 2014). In addition, the high cognitive requirements of the PG and CG also had a significant impact on processing speed. The results of this experiment are similar to previous research (Ji et al., 2017), and the combined motion with higher cognitive requirements has broader motor benefits for inhibition, which supports the research hypothesis. The results of physical fitness showed that the cardiorespiratory endurance and dynamic balance of the PG were better than those of the HG and CG.

This experiment used short-term interventions to explore the effects of different cognitive requirements on inhibition. The required pattern of exercise combined with cognition for the physical exercise tapping high-level cognitive functions condition led to the most significant improvement in cognitive function, not only in the executive conditions but also in perception speed. This is consistent with our early research (Ji et al., 2019), and it can be speculated that the effect of physical exercise tapping high-level cognitive functions on cognitive function may be related to the effects of different exercise patterns on brain activation, especially different intensities of prefrontal activation. This result also supports the theory of adaptive ability, and the result that physical exercise combined with cognitive training showed a more significant improvement compared to the other three groups. A study with 138 volunteers by León et al. (2015) found that physical and cognitive exercise programmes produced significant positive effects, with improvements in both simple and selective responses. Curlik 2nd and Shors (2013) believe that the physiological responses obtained through physical and cognitive exercise programmes seem to require neurogenesis and keep new cells alive over time. Therefore, Budde et al. (2008) believe that a complex task can effectively promote cognitive neural networks. At the same time, in this experiment, cognitive training also had a significant effect on cognitive function, which is different from the results of our prior research (Ji et al., 2019). It is speculated that the influence of cognitive training on cognitive function may not depend entirely on the influence of brain activation. In addition to examining the effect of exercise on cognitive function, our study also examined the effects of different intervention times. We found that the effect of physical exercise on inhibitory function occurred in the 12th week of the test, and the effects of cognitive training and exercise requiring high cognition levels on cognitive function appeared in the 8th week. This is consistent with previous findings that the 6-week training plan used by Owen et al. (2010) can significantly improve cognitive functions, such as reasoning, memory, planning, visual spatial skills, and attention. In a study by Theill et al. (2013), cognitive training interventions were similar to cognitive interventions. The effect of physical exercise on the inhibition function was set at 12 weeks. Therefore, exercise requiring high cognitive levels is more efficient than physical exercise in improving cognitive function, and the effect is better than simple cognitive training.

The current studyexamined the effects of various activities on cognitive function and physical fitness. Physical fitness (i.e., cardiopulmonary endurance and muscle strength) also included exercise adaptability (i.e., flexibility, speed, balance, and good coordination), and exercise can improve the level of fitness (Brent, 2009). On one hand, we could examine the improvement of physical fitness after different activities, while on the other hand, we could examine whether the improvement of the inhibitory function after the exercise with high cognitive requirements sacrifices improvements in physical fitness. The results showed that the physical fitness indices indicating the dynamic balance, cardiorespiratory endurance, and physical activity level were significantly better than the HG in the 12 -week post-test. The times of the TUG and mTUG tests were also significantly shorter than that of the HG, which suggests that exercise requires a longer intervention time for the improvement of physical fitness compared with inhibition. At the same time, the improvement of cognitive function by physical exercise tapping high-level cognitive functions does not affect the impact of exercise on the fitness level due to increased cognitive re- 
quirements (Hiyamizu et al., 2012). The results of the relevant studies are consistent, and different cognitive requirements can result in the benefits of physical fitness (Hiyamizu et al., 2012). In our experiment, high cognition required that the CG exhibit a better dual-task function than the PG, which is consistent with previous experimental and research hypotheses. High cognitive motion requires attention to the allocation of exercise to simultaneously accomplish two tasks, so the improvement of dual-task processing is more significant.

\section{CONCLUSION}

Physical exercise tapping high-level cognitive functions and cognitive training can improve executive function more quickly than physical exercise alone. The effects of physical exercise tapping high-level cognitive functions and physical exercise alone showed no significant differences in physical fitness, but they were both better than cognitive training alone, especially regarding dynamic balance effects.

\section{REFERENCES}

Albinet, C. T., Aboudest, A., André, N., \& Audiffren, M. (2016). Executive functions improvement following a 5-month aquaerobics program in older adults: Role of cardiac vagal control in inhibition performance. Biological Psychology, 115,

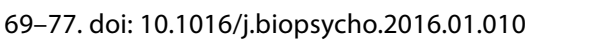

Bohnen, N., Twijnstra, A., \& Jolles, J. (1992). Performance in the Stroop color word test in relationship to the persistence of symptoms following mild head injury. Acta Neurologica Scandinavica, 85, 116-121. doi: 10.1111/j.1600-0404.1992.

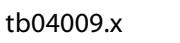

Boucard, G. K., Albinet, C. T., Bugaiska, A., Bouquet, C. A., Clarys, D., \& Audiffren, M. (2012). Impact of physical activity on executive functions in aging: A selective effect on inhibition among old adults. Journal of Sport \& Exercise Psychology, 34, 808-827. doi: 10.1123/jsep.34.6.808 الس

Brent, S. E. (2010). ACSM's health-related physical fitness assessment manual. Wolters Kluwer Health.

Budde, H., Voelcker-Rehage, C., Pietraßyk-Kendziorra, S., Ribeiro, P., \& Tidow, G. (2008). Acute coordinative exercise improves attentional performance in adolescents. Neuroscience Letters, 441, 219-223. doi: 10.1016/j.neulet.2008.06.024 المالسلس

Burr, J. F., Bredin, S. S., Faktor, M. D., \& Warburton, D. E. (2011). The 6-minute walk test as a predictor of objectively measured aerobic fitness in healthy working-aged adults. The Physician and Sportsmedicine, 39, 133-139. doi: 10.3810/psm.2011.05.1904 الفيلسلس

Camarri, B., Eastwood, P. R., Cecins, N. M., Thompson, P. J., \& Jenkins, S. (2006). Six minute walk distance in healthy subjects aged 55-75 years. Respiratory Medicine, 100, 658-665. doi: 10.1016/j.rmed.2005.08.003 الس

Clark, R. A., Bryant, A. L., Pua, Y., McCrory, P., Bennell, K., \& Hunt, M. (2010). Validity and reliability of the Nintendo Wii Balance Board for assessment of standing balance. Gait \& Posture, 31 , 307-310. doi: 10.1016/j.gaitpost.2009.11.012 المالسلسلة
Cockrell, J. R., \& Folstein, M. F. (1988). Mini-Mental State Examination (MMSE). Psychopharmacological Bulletin, 24, 689-692. البلسلسلسا.

Curlik 2nd, D., \& Shors, T. (2013). Training your brain: Do mental and physical (MAP) training enhance cognition through the process of neurogenesis in the hippocampus? Neuropharmacology, 64, 506-514. doi: 10.1016/j.neuropharm.2012.07.027 السلسلس

Dupuy, O., Gauthier, C. J., Fraser, S. A., Laurence, D. C., Michèle, D., Said, M., . . Louis, B. (2015). Higher levels of cardiovascular fitness are associated with better executive function and prefrontal oxygenation in younger and older women. Frontiers in Human Neuroscience, 9, 66. doi: 10.3389/fnhum.2015.00066 |لسلسلس

Fabre, C., Chamari, K., Mucci, P., Massé-Biron, J., \& Préfaut, C. (2002). Improvement of cognitive function by mental and/ or individualized aerobic training in healthy elderly subjects. International Journal of Sports Medicine, 23, 415-421. doi: 10.1055/s-2002-33735 الس الس

Hallal, P. C., \& Victora, C. G. (2004). Reliability and validity of the International Physical Activity Questionnaire (IPAQ). Medicine \& Science in Sports \& Exercise, 36, 556. doi: 10.1249/01. MSS.0000117161.66394.07 سلس

Hartman, M., \& Hasher, L. (1991). Aging and suppression: Memory for previously relevant information. Psychology \& Aging, 6, 587-594. doi: 10.1037/0882-7974.6.4.587 الفيلس

Hiyamizu, M., Morioka, S., Shomoto, K., \& Shimada, T. (2012). Effects of dual task balance training on dual task performance in elderly people: a randomized controlled trial. Clinical Rehabilitation, 26, 58-67. doi: 10.1177/0269215510394222 المالسلس Huang, C.-J., Lin, P.-C., Hung, C.-L., Chang, Y.-K., \& Hung, T.-M. (2014). Type of physical exercise and inhibitory function in older adults: An event-related potential study. Psychology of Sport and Exercise, 15, 205-211. doi: 10.1016/j.psychsport.2013.11.005 السلسلس

Hung, N. M., \& Andreas, K. (2012). A randomized controlled trial of Tai chi for balance, sleep quality and cognitive performance in elderly Vietnamese. Clinical Interventions in Aging, 7, 185-190. doi: 10.2147/CIA.S32600 سلس

Ji, Z, Feng, T., Mei, L., Li, A., \& Zhang, C. (2019). Influence of acute combined physical and cognitive exercise on cognitive function: An NIRS study. PeerJ, 7, e7418. السلسلس

Ji, Z., Li, A., Feng, T., Liu, X., \& Zhang, C. (2017). The benefits of Tai Chi and brisk walking for cognitive function and fitness in older adults. PeerJ, 5, e3943.

Kim, T. H., Pascualleone, J., Johnson, J., \& Tamim, H. (2016). The mental-attention Tai Chi effect with older adults. BMC Psychology, 4, 1-15. doi: 10.1186/s40359-016-0137-0 السلسلس

Law, L. L., Barnett, F., Yau, M. K., \& Gray, M. A. (2014). Effects of combined cognitive and exercise interventions on cognition in older adults with and without cognitive impairment: A systematic review. Ageing Research Reviews, 15, 61-75. doi: 
10.1016/j.arr.2014.02.008

León, J., Ureña, A., Bolaños, M. J., Bilbao, A., \& Oña, A. (2015). A combination of physical and cognitive exercise improves reaction time in persons 61-84 years old. Journal of Aging \& Physical Activity, 23, 72-77. doi: 10.1123/JAPA.2012-0313 السلسلس

Liou, Y. M., Jwo, C. J., Yao, K. G., Chiang, L. C., \& Huang, L. H. (2008). Selection of appropriate Chinese terms to represent intensity and types of physical activity terms for use in the Taiwan version of IPAQ. Journal of Nursing Research, 16, 252-263. doi:

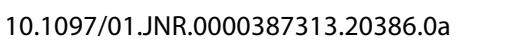

Man, D. W., Tsang, W. W., \& Hui-Chan, C. W. (2010). Do older t'ai chi practitioners have better attention and memory function? Journal of Alternative \& Complementary Medicine, 16, 12591264. doi: 10.1089/acm.2009.0462 السلسلسلس

Matthews, M. M., \& Williams, H. G. (2008). Can Tai chi enhance cognitive vitality? A preliminary study of cognitive executive control in older adults after a Tai chi intervention. Journal of

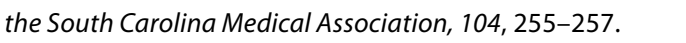

Muir, S. W., Gopaul, K., \& Odasso, M. M. M. (2012). The role of cognitive impairment in fall risk among older adults: A systematic review and meta-analysis. Age \& Ageing, 41, 299-308. doi:

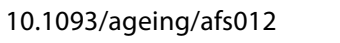

Owen, A. M., Hampshire, A., Grahn, J. A., Stenton, R., Dajani, S., Burns, A. S., . . Ballard, C. G. (2010). Putting brain training to

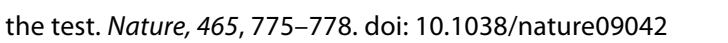

Pichierri, G., Wolf, P., Murer, K., \& Bruin, E. D. D. (2011). Cognitive and cognitive-motor interventions affecting physical functioning: a systematic review. BMC Geriatrics, 11, 29. doi: 10.1186/1471-2318-11-29 المالسلسلس

Porciuncula, F. S., Rao, A. K., \& Mclsaac, T. L. (2016). Aging-related decrements during specific phases of the dual-task Timed Up-and-Go test. Aging Clinical and Experimental Research, 28,

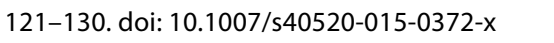

Reid-Arndt, S. A., Matsuda, S., \& Cox, C. R. (2012). Tai Chi effects on neuropsychological, emotional, and physical functioning following cancer treatment: A pilot study. Complementary Therapies in Clinical Practice, 18, 26-30. doi: 10.1016/j. ctcp.2011.02.005 المبلس

Gates, N. J., Sachdev, P. S., Fiatarone Singh. M. A., \& Valenzuela, M. (2011). Cognitive and memory training in adults at risk of dementia: A systematic review. BMC Geriatrics, 11, 55. doi: 10.1186/1471-2318-11-55 الس السلسل

Shinya, U., \& Ryuta, K. (2008). Reading and solving arithmetic problems improves cognitive functions of normal aged people: a randomized controlled study. $A G E, 30,21-29$. doi: 10.1007/s11357-007-9044-x السلسلسلس

Shumway-Cook, A., Brauer, S., \& Woollacott, M. (2000). Predicting the probability for falls in community-dwelling older adults using the Timed Up \& Go Test. Physical Therapy, 80, 896-903. doi: 10.1093/ptj/80.9.896 السلسلس

Smith, P. J., Blumenthal, J. A., Hoffman, B. M., Cooper, H., Strauman, T. A., Welsh-Bohmer, K., ... Sherwood, A. (2010). Aerobic exercise and neurocognitive performance: A metaanalytic review of randomized controlled trials. Psychosomatic Medicine, 72, 239-252. doi: 10.1097/PSY.0b013e3181d14633 Wل

Theill, N., Schumacher, V., Adelsberger, R., Martin, M., \& Jäncke, L. (2013). Effects of simultaneously performed cognitive and physical training in older adults. BMC Neuroscience, 14, 103.

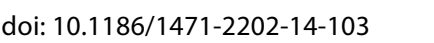

Zhu, X., Yin, S., Lang, M., He, R., \& Li, J. (2016). The more the better? A meta-analysis on effects of combined cognitive and physical intervention on cognition in healthy older adults. Ageing Research Reviews, 31, 67-79. doi: 10.1016/j.arr.2016.07.003 المبلسلس 